\title{
Ruthenium-Catalyzed Tandem Cross-Metathesis/Wittig Olefination: Generation of Conjugated Dienoic Esters from Terminal Olefins
}

\author{
Ryan P. Murelli and Marc L. Snapper* \\ Department of Chemistry, Merkert Chemistry Center, Boston College, 2609 Beacon Street, Chestnut Hill, Massachusetts 02467-3860 \\ marc.snapper@bc.edu
}

\section{Supporting Information}

Table of Contents

General Information

Page

Experimental Procedures and Spectral Data

S2-S5

(2E,4E)-Ethyl 4-methylundeca-2,4-dienoate (8a)

(2E,4E)-t-Butyl 4-methylundeca-2,4-dienoate $\mathbf{( 8 b )}$

(2E,4E)-Ethyl 13-(tert-butyldimethylsilyloxy)-4-methyltrideca-2,4-dienoate (8c)

S2-S3

(2E,4E)-Ethyl 10-(benzyloxy)-4-methyldeca-2,4-dienoate (8d)

(2E,4E)-Ethyl 9-acetoxy-4-methylnona-2,4-dienoate (8e)

(2E,4E)-t-Butyl undeca-2,4-dienoate $(\mathbf{8 f})$

(2E,4E)-Ethyl 6-(2-oxocyclohexyl)hexa-2,4-dienoate $(\mathbf{8 g})$

(4E)-Ethyl 3-methyl-5-phenylpenta-2,4-dienoate (9) and stereoisomer (9b)

\section{General Information}

Unless otherwise stated, all reactions are carried out in flame-dried vials with Teflon-sealed screwcaps. For slow addition at refluxing temperatures, the vial is fitted with rubber septa pierced with a hole adequate for a cold-finger. Starting materials and reagents were purchased from chemical suppliers and used without further purification except the following: $\mathrm{CH}_{2} \mathrm{Cl}_{2}$ and toluene were dried on alumina columns using a solvent dispensing system; methacrolein was distilled over $\mathrm{CaSO}_{4}$, stored at $-20{ }^{\circ} \mathrm{C}$, and used within one month of distillation.

Infrared (IR) spectra bands are characterized as broad (br), strong (s), medium (m) and weak (w).

${ }^{1}$ H-NMR chemical shifts are reported with the solvent as the internal standard $\left(\mathrm{CHCl}_{3} \delta 7.26 \mathrm{ppm}\right)$. Data are reported as follows: chemical shift, multiplicity $(\mathrm{s}=$ singlet, $\mathrm{d}=$ doublet, $\mathrm{t}=$ triplet, $\mathrm{q}=\mathrm{quartet}$, $\mathrm{br}=$ broad, $\mathrm{m}=$ multiplet), coupling constants $(\mathrm{Hz})$, and integration. ${ }^{13} \mathbf{C}-\mathbf{N M R}$ chemical shifts are reported in ppm with the solvent as an internal reference $\left(\mathrm{CDCl}_{3} \delta 77.2 \mathrm{ppm}\right)$. Reactions monitored by Gas Chromatography used a $100 \%$ methyl polysiloxane column. 


\section{Experimental Procedures}

Procedure A: (2E,4E)-Ethyl 4-methylundeca-2,4-dienoate (8a) ${ }^{1}$. A solution of methacrolein $(20 \mu \mathrm{L}$,<smiles>CCCCCC/C=C(C)/C=C/C=O</smiles>

deemed complete by ${ }^{1} \mathrm{H}$ NMR. Triphenylphosphine $(126 \mathrm{mg}, 0.484 \mathrm{mmol}$ ) was then added to the reaction, a condenser was connected, and ethyl diazoacetate $(81 \mu \mathrm{L}, 0.73 \mathrm{mmol})$ dissolved in $\mathrm{CH}_{2} \mathrm{Cl}_{2}(500$ $\mu \mathrm{L}$ ) was added slowly over $4 \mathrm{~h}$ at reflux. After the addition was complete, the vial was again sealed and heated to $60{ }^{\circ} \mathrm{C}$ for $4-8 \mathrm{~h}$. Concentration and silica gel chromatography (20:1 hexanes: diethyl ether) afforded the desired product 8a as a clear oil (42 mg, 82\% yield). FTIR ( $\mathrm{NaCl}$, thin film) 2968(s), 2864(m), 1705(s), 1620(m), 1390(m), 1319(m), 1149(s), 972(m) cm ${ }^{-1} .{ }^{1}$ H-NMR (400 MHz, $\left.\mathrm{CDCl}_{3}\right): \delta$ 0.82-0.90 (m, 3H), 1.20-1.34 (m, 7H), 1.44-1.34 (m, 2H), 1.55-1.44 (m, 2H), 1.74 (s, 3H), 2.16 (q, $J=7.3$ $\mathrm{Hz}, 2 \mathrm{H}), 4.18$ (q, $J=7.3 \mathrm{~Hz}, 2 \mathrm{H}), 5.75$ (d, $J=15.4 \mathrm{~Hz}, 1 \mathrm{H}), 5.87$ (t, $J=7.3 \mathrm{~Hz}, 1 \mathrm{H}), 7.29$ (d, $J=15.4 \mathrm{~Hz}$, 1H). ${ }^{13}$ C-NMR (100 MHz, $\left.\mathrm{CDCl}_{3}\right): \delta$ 167.6, 149.7, 142.5, 132.8, 115.5, 60.4, 32.0, 29.3, 29.1, 29.1, 22.9, $14.7,14.4,12.5$.

(2E,4E)-t-Butyl 4-methylundeca-2,4-dienoate (8b). Following procedure A, replacing ethyl diazoacetate $\mathrm{CO}_{2} \mathrm{t}-\mathrm{Bu}$ with t-butyl diazoacetate $(101 \mu \mathrm{L}, 0.726 \mathrm{mmol})$ and purifying with silica gel chromatography (20:1 hexanes:diethyl ether) afforded a clear oil $\mathbf{8 b}(53 \mathrm{mg}, 87 \%$ yield $)$. FTIR $(\mathrm{NaCl}$, thin 0.79-0.92 (t, $J=6.6 \mathrm{~Hz}, 3 \mathrm{H}), 1.20-1.33(\mathrm{~m}, 6 \mathrm{H}), 1.35-1.45(\mathrm{~m}, 2 \mathrm{H}), 1.49(\mathrm{~s}, 9 \mathrm{H}), 1.74(\mathrm{~s}, 3 \mathrm{H}), 2.17$ (q, $J=$ $7.3 \mathrm{~Hz}, 2 \mathrm{H}), 5.70(\mathrm{~d}, J=16.1 \mathrm{~Hz}, 1 \mathrm{H}), 5.85(\mathrm{t}, J=7.3 \mathrm{~Hz}, 1 \mathrm{H}), 7.21$ (d, $J=16.1 \mathrm{~Hz}, 1 \mathrm{H}) .{ }^{13} \mathbf{C}-\mathbf{N M R}(100$ $\left.\mathrm{MHz}, \mathrm{CDCl}_{3}\right): \delta 167.0,148.7,141.7,132.7,117.4,80.1,32.0,29.4,29.3,29.1,28.5,22.9,14.4,12.5$. HRMS (EI+) $m / z$ calcd for $\mathrm{C}_{16} \mathrm{H}_{28} \mathrm{O}_{2}: 252.2089$. Mass Found: 252.2091 .

(2E,4E)-Ethyl 13-(t-butyldimethylsilyloxy)-4-methyltrideca-2,4-dienoate (8c). Following procedure A, $\begin{aligned} & \text { replacing 1-octene with t-butyl(dec-9-enyloxy)di- } \\ & \text { methylsilane }(130 \mathrm{mg}, 0.484 \mathrm{mmol}) \text { and purifying }\end{aligned}$
$\mathrm{CO}_{2} \mathrm{Et} \quad \begin{aligned} & \mathrm{me} \text { with silica gel chromatography (10:1 hexanes:di- }\end{aligned}$ ethyl ether) afforded a clear oil 8c (69 mg, 75\% yield). FTIR ( $\mathrm{NaCl}$, thin film) 3047(m), 2969(s), 2870(s), 1712(s), 1646(m), 1477(w), 1169(s), 842(m) cm ${ }^{-1} .{ }^{1}$ H-NMR (400 MHz, $\left.\mathrm{CDCl}_{3}\right): \delta 0.03(\mathrm{~s}, 6 \mathrm{H}), 0.88$ (s, $9 \mathrm{H}), 1.20-1.34(\mathrm{~m}, 11 \mathrm{H}), 1.44-1.34(\mathrm{~m}, 2 \mathrm{H}), 1.55-1.44(\mathrm{~m}, 2 \mathrm{H}), 1.75(\mathrm{~s}, 3 \mathrm{H}), 2.18(\mathrm{q}, J=7.3 \mathrm{~Hz}, 2 \mathrm{H}), 3.58$ (t, $J=6.6 \mathrm{~Hz}, 2 \mathrm{H}), 4.20(\mathrm{q}, J=7.3 \mathrm{~Hz}, 2 \mathrm{H}), 5.77$ (d, $J=15.4 \mathrm{~Hz}, 1 \mathrm{H}), 5.89$ (t, $J=7.3 \mathrm{~Hz}, 1 \mathrm{H}), 7.31$ (d, $J=$

${ }^{1}$ For an earlier citation describing this molecule, see: Kukovinets, O. S.; Zainullin, R. A.; Kasradze, V. G.; Burov, V. N.; Mokrousova, E. P.; Odinokov, V. N.; Tolstikov, G. A. Agrokhimiya 1999, 9, 60-63. 
$15.4 \mathrm{~Hz}, 1 \mathrm{H}) .{ }^{13} \mathbf{C}-\mathbf{N M R}\left(100 \mathrm{MHz}, \mathrm{CDCl}_{3}\right): \delta 167.5,149.7,142.4,132.7,115.5,63.5,60.3,33.1,29.7$, 29.6, 29.5, 29.3, 29.1, 26.8, 22.1, 18.7, 14.7, 12.4, -4.9. HRMS (TOF MS ES+) $m / z$ calcd for $\mathrm{C}_{22} \mathrm{H}_{43} \mathrm{O}_{3} \mathrm{Si}$ : 383.2981. Mass Found: 383.2979.

(2E,4E)-Ethyl 10-(benzyloxy)-4-methyldeca-2,4-dienoate (8d). Following procedure A, replacing 1-

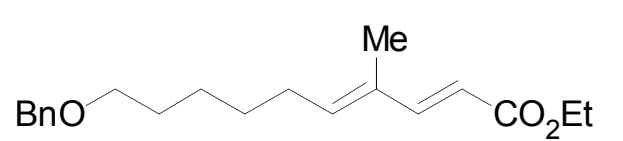
octene with ((hept-6-enyloxy)methyl)benzene (98 mg, 0.48 mmol), and purifying with silica gel chromatography (10:1 hexanes: diethyl ether) afforded a clear oil $\mathbf{8 d}(55 \mathrm{mg}, 72 \%$ yield). FTIR ( $\mathrm{NaCl}$, thin film) 3040(s), 2936(m), 2857(m), 1718(s), 1273(w), 1280(m), 1182(m) $\mathrm{cm}^{-1}$. ${ }^{1}$ H-NMR $\left(400 \mathrm{MHz}, \mathrm{CDCl}_{3}\right): \delta 1.26-1.33(\mathrm{~m}, 2 \mathrm{H}), 1.30(\mathrm{t}, J=7.3 \mathrm{~Hz}, 3 \mathrm{H}), 1.36-1.47(\mathrm{~m}, 4 \mathrm{H}), 1.59-1.66$ (m, 2H), 1.76 (s, 3H), 2.20 (q, $J=7.3 \mathrm{~Hz}, 2 \mathrm{H}), 3.39$ (t, $J=6.6 \mathrm{~Hz}, 2 \mathrm{H}), 4.20$ (q, $J=7.3 \mathrm{~Hz}, 2 \mathrm{H}), 4.50$ (s, 2H), $5.78(\mathrm{~d}, J=15.4 \mathrm{~Hz}, 1 \mathrm{H}), 5.88(\mathrm{t}, J=7.3 \mathrm{~Hz}, 1 \mathrm{H}), 7.20-7.44(\mathrm{~m}, 6 \mathrm{H}) .{ }^{13} \mathbf{C}-\mathbf{N M R}\left(100 \mathrm{MHz}, \mathrm{CDCl}_{3}\right)$ :

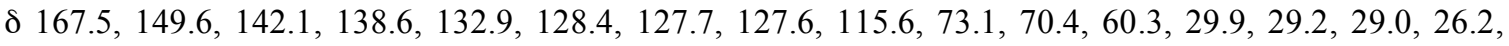
14.7, 12.4. HRMS (TOF MS ES+) $m / z$ calcd for $\mathrm{C}_{20} \mathrm{H}_{28} \mathrm{O}_{3} \mathrm{Na}$ : 339.1936. Mass Found: 339.1929.

Procedure B: (2E,4E)-Ethyl 9-acetoxy-4-methylnona-2,4-dienoate (8e). A solution of methacrolein<smiles>CCOC(=O)C=CC(C)=CCCCCOC(C)=O</smiles>
$(110 \mu \mathrm{L}, 1.21 \mathrm{mmol})$, hexenyl acetate $(54 \mu \mathrm{L}, 0.34 \mathrm{mmol})$, and Grubbs' vinylidene catalyst IV (14 $\mathrm{mg}, 0.017 \mathrm{mmol})$ in $\mathrm{CH}_{2} \mathrm{Cl}_{2}(600 \mu \mathrm{L})$ was heated in a sealed vial at $60{ }^{\circ} \mathrm{C}$ for $3-4$ $h$, or until deemed complete by ${ }^{1} \mathrm{H}$ NMR. The vial was unscrewed, and the solvent was allowed to evaporate for $0.5 \mathrm{~h}$, until an aliquot revealed no remaining methacrolein by ${ }^{1} \mathrm{H}$ NMR. $\mathrm{CH}_{2} \mathrm{Cl}_{2}(600 \mu \mathrm{L})$ and triphenylphosphine $(175 \mathrm{mg}, 0.670 \mathrm{mmol})$ were then added to the reaction, a condenser was connected, and ethyl diazoacetate $(112 \mu \mathrm{L}, 1.01 \mathrm{mmol})$ dissolved in $\mathrm{CH}_{2} \mathrm{Cl}_{2}(600 \mu \mathrm{L})$ was added slowly over $4 \mathrm{~h}$ at reflux. After the addition was complete, the vial was again sealed and heated to $60{ }^{\circ} \mathrm{C}$ for $4-8 \mathrm{~h}$. Concentration and silica gel chromatography (3:1 hexanes: diethyl ether) afforded the desired product $8 \mathrm{e}$ as a clear oil $(71 \mathrm{mg}, 86 \%$ yield). FTIR ( $\mathrm{NaCl}$, thin film) 3093(s), 3040(s), 1751(m), 1712(m), 1228(m) $\mathrm{cm}^{-1} .{ }^{1}$ H-NMR (400 MHz, $\left.\mathrm{CDCl}_{3}\right): \delta 1.29$ (t, $\left.J=6.6,3 \mathrm{H}\right), 1.49$ (m, 2H), $1.64(\mathrm{~m}, 2 \mathrm{H}), 1.77(\mathrm{~s}, 3 \mathrm{H}), 2.05(\mathrm{~s}, 3 \mathrm{H}), 2.32(\mathrm{q}, J=7.3 \mathrm{~Hz}, 2 \mathrm{H}), 4.06(\mathrm{t}, J=6.6 \mathrm{~Hz}, 2 \mathrm{H}), 4.20(\mathrm{q}, J=$ $7.3 \mathrm{~Hz}, 2 \mathrm{H}), 5.79$ (d, $J=16.1 \mathrm{~Hz}, 1 \mathrm{H}), 5.87$ (t, $J=7.3 \mathrm{~Hz}, 1 \mathrm{H}), 7.31$ (d, $J=16.1 \mathrm{~Hz}, 1 \mathrm{H}) .{ }^{13} \mathbf{C}-\mathbf{N M R}(100$ $\left.\mathrm{MHz}, \mathrm{CDCl}_{3}\right) \delta 171.1,167.5,149.3,141.6,133.3,115.9,64.4,60.4,28.6,28.5,25.7,21.3,14.6,12.5$. HRMS (TOF MS ES+) $m / z$ calcd for $\mathrm{C}_{14} \mathrm{H}_{22} \mathrm{O}_{4} \mathrm{Na}: 277.1416$. Mass Found: 277.1427.

Procedure C: (2E,4E)-t-Butyl undeca-2,4-dienoate (8f). A solution of acrolein (112 $\mu \mathrm{L}, 1.68 \mathrm{mmol})$, $\mathrm{CO}_{2} \mathrm{t}-\mathrm{Bu}$ octene (54 $\mu \mathrm{L}, 0.34 \mathrm{mmol})$, and Hoveyda-Grubbs' II (III) $(10 \mathrm{mg}, 0.017 \mathrm{mmol})$ in $\mathrm{CH}_{2} \mathrm{Cl}_{2}(600 \mu \mathrm{L})$ was heated in a sealed vial at $60{ }^{\circ} \mathrm{C}$ for $32 \mathrm{~h}$, or until deemed complete by thin layer chromatography. The vial was unscrewed, and the solvent was allowed to evaporate for $0.5 \mathrm{~h}$, until aliquot revealed no remaining acrolein 
by ${ }^{1} \mathrm{H}-\mathrm{NMR} . \quad \mathrm{CH}_{2} \mathrm{Cl}_{2}(600 \mu \mathrm{L})$ and triphenylphosphine $(175 \mathrm{mg}, 0.670 \mathrm{mmol})$ were then added to the reaction, a condenser was connected, and $t$-butyl diazoacetate $(140 \mu \mathrm{L}, 1.01 \mathrm{mmol})$ dissolved in $\mathrm{CH}_{2} \mathrm{Cl}_{2}$ $(600 \mu \mathrm{L})$ was added slowly over $4 \mathrm{~h}$ at reflux. After the addition was complete, the vial was again sealed and heated to $60{ }^{\circ} \mathrm{C}$ for $4-8 \mathrm{~h}$. Concentration and silica gel chromatography (50:1 hexanes: diethyl ether) afforded the desired product $\mathbf{8 f}$ as a clear oil (47 mg, 59\% yield). FTIR ( $\mathrm{NaCl}$, thin film) $3034(\mathrm{~m})$, 2923(s), 2864(w), 1705(s), 1653(m), 1372(m), 1136(s), 998(m) cm ${ }^{-1} .{ }^{1} \mathbf{H}-\mathbf{N M R}\left(400 \mathrm{MHz}, \mathrm{CDCl}_{3}\right): \delta 0.87$ (t, $J=7.3 \mathrm{~Hz}, 3 \mathrm{H}), 1.20-1.40(\mathrm{~m}, 8 \mathrm{H}), 1.47$ (s, 9H), 2.14 (q, $J=7.3 \mathrm{~Hz}, 2 \mathrm{H}), 5.70(\mathrm{~d}, J=15.4 \mathrm{~Hz}, 1 \mathrm{H})$, 6.00-6.25 (m, 2H), 7.15 (dd, $J=15.4,10.3 \mathrm{~Hz}, 1 \mathrm{H}) .{ }^{13} \mathbf{C}-\mathbf{N M R}\left(100 \mathrm{MHz}, \mathrm{CDCl}_{3}\right)$ : $\delta$ 166.6, 144.1, 144.0, 128.4 121.2, 80.1, 33.2, 31.9, 29.1, 29.0, 28.5, 22.9, 14.4. HRMS (EI+) $m / z$ calcd for $\mathrm{C}_{15} \mathrm{H}_{26} \mathrm{O}_{2}: 238.1933$. Mass Found: 238.1930.

(2E,4E)-Ethyl 6-(2-oxocyclohexyl)hexa-2,4-dienoate (8g). Following procedure C, replacing t-butyl

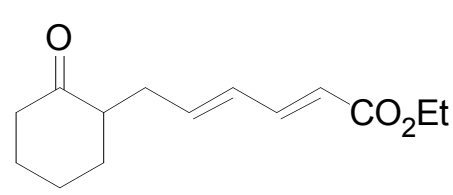
diazoacetate and 1-octene with ethyl diazoacetates ( $81 \mu \mathrm{L}, 0.726 \mathrm{mmol})$ and 97\% 2-allylcyclohexanone (50 $\mu \mathrm{L}, 0.325 \mathrm{mmol})$ respectively, and purifying with silica gel chromatography (10:1 hexanes:diethyl ether) afforded a clear oil $\mathbf{8 g}(50 \mathrm{mg}$, 63\% yield). FTIR ( $\mathrm{NaCl}$, thin film) 3047(m), 2942(m), 2870(m), 1712(s), 1640(m), 1247(m), 1129(m) cm ${ }^{-1} .{ }^{1} \mathbf{H}-\mathbf{N M R}\left(400 \mathrm{MHz}, \mathrm{CDCl}_{3}\right): \delta$ 1.00-1.50 (m, 2H), 1.26 (t, $J=7.3 \mathrm{~Hz}, 2 \mathrm{H}), 1.75-1.95$ (m, 2H), 1.95-2.21 (m, 2H), 2.21-2.47 (m, 3H), 2.60 $(\mathrm{dt}, J=14.6,5.8 \mathrm{~Hz} 1 \mathrm{H}), 4.17$ (q, $J=7.3 \mathrm{~Hz}, 2 \mathrm{H}), 5.77(\mathrm{~d}, J=15.4 \mathrm{~Hz}, 1 \mathrm{H}), 6.06(\mathrm{dt}, J=15.4,7.3 \mathrm{~Hz}$, 1H), $6.15(\mathrm{dd}, J=15.4,11.0 \mathrm{~Hz}, 1 \mathrm{H}), 7.18-7.24$ (dd, $J=15.4,10.3 \mathrm{~Hz}, 1 \mathrm{H}) .{ }^{13} \mathbf{C}-\mathbf{N M R}\left(100 \mathrm{MHz}, \mathrm{CDCl}_{3}\right)$ : $\delta$ 2 211.6, 167.1 144.6, 141.8, 130.1, 119.8, 60.4, 50.5, 42.3, 33.9, 33.2, 28.2, 25.4, 14.6. HRMS (TOF MS ES+) $m / z$ calcd for $\mathrm{C}_{14} \mathrm{H}_{20} \mathrm{O}_{3} \mathrm{Na}$ : 259.1310. Mass Found: 259.1317 .

Procedure D: (4E)-Ethyl 3-methyl-5-phenylpenta-2,4-dienoate (9) and stereoisomer (9b) A solution of

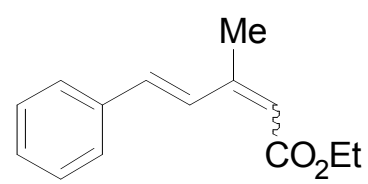
methyl vinyl ketone $(20 \mu \mathrm{L}, 0.24 \mathrm{mmol})$, styrene $(80 \mu \mathrm{L}, 0.66 \mathrm{mmol})$, and Grubbs' vinylidene catalyst (IV) $(13 \mathrm{mg}, 0.016 \mathrm{mmol})$ in toluene $(1.2 \mathrm{~mL})$ was heated in a sealed vial at $50{ }^{\circ} \mathrm{C}$ for $10-12 \mathrm{~h}$, or until deemed complete by ${ }^{1} \mathrm{H}$ NMR. Triphenylphosphine (345 mg, $1.32 \mathrm{mmol})$ and benzoic acid $(20 \mathrm{mg}$, $0.14 \mathrm{mmol})$ were then added to the reaction. Ethyl diazoacetate $(140 \mu \mathrm{L}, 1.01 \mathrm{mmol})$ was added slowly over $10 \mathrm{~h}$ at $90^{\circ} \mathrm{C}$, and the reaction was held at this temperature for another $20 \mathrm{~h}$. Concentration and silica gel chromatography (20:1 hexanes:diethyl ether) afforded the desired products $\mathbf{9}$ and $\mathbf{9 b}$ as a mixture of cis/trans isomers (37 mg, 71\% yield). 9: ${ }^{1} \mathbf{H}-\mathbf{N M R}\left(400 \mathrm{MHz}, \mathrm{CDCl}_{3}\right): \delta 8.42(\mathrm{~d}, J=16.8 \mathrm{~Hz}, 1 \mathrm{H}), 7.22-$ 7.62 (m, aromatic), $6.92(\mathrm{~d}, J=16.1 \mathrm{~Hz}, 1 \mathrm{H}), 5.75(\mathrm{~s}, 1 \mathrm{H}), 4.20(\mathrm{q}, J=7.3 \mathrm{~Hz}, 2 \mathrm{H}), 2.13(\mathrm{~s}, 3 \mathrm{H}), 1.32$ (t, $J$ $=7.3 \mathrm{~Hz}, 2 \mathrm{H}) .9 \mathrm{~b}: 7.22-7.62(\mathrm{~m}$, aromatic), $6.94(\mathrm{~d}, J=16.1 \mathrm{~Hz}, 1 \mathrm{H}), 6.81(\mathrm{~d}, J=16.1 \mathrm{~Hz}, 1 \mathrm{H}), 5.91(\mathrm{~s}$, $1 \mathrm{H}), 4.20$ (q, $J=7.3 \mathrm{~Hz}, 2 \mathrm{H}), 2.41(\mathrm{~s}, 3 \mathrm{H}), 1.32(\mathrm{t}, J=7.3 \mathrm{~Hz}, 2 \mathrm{H})$. E/Z ratio was determined by ${ }^{I} H \mathrm{NMR}$ 
integration of the methyl groups (2.13 ppm vs. $2.41 \mathrm{ppm})$, and the configuration is determined by comparison to known data. ${ }^{2}$

${ }^{2}$ Lee, M.; Chen, Y.; Zhang, X. P. Organometallics, 2003, 22, 4905-4909. 


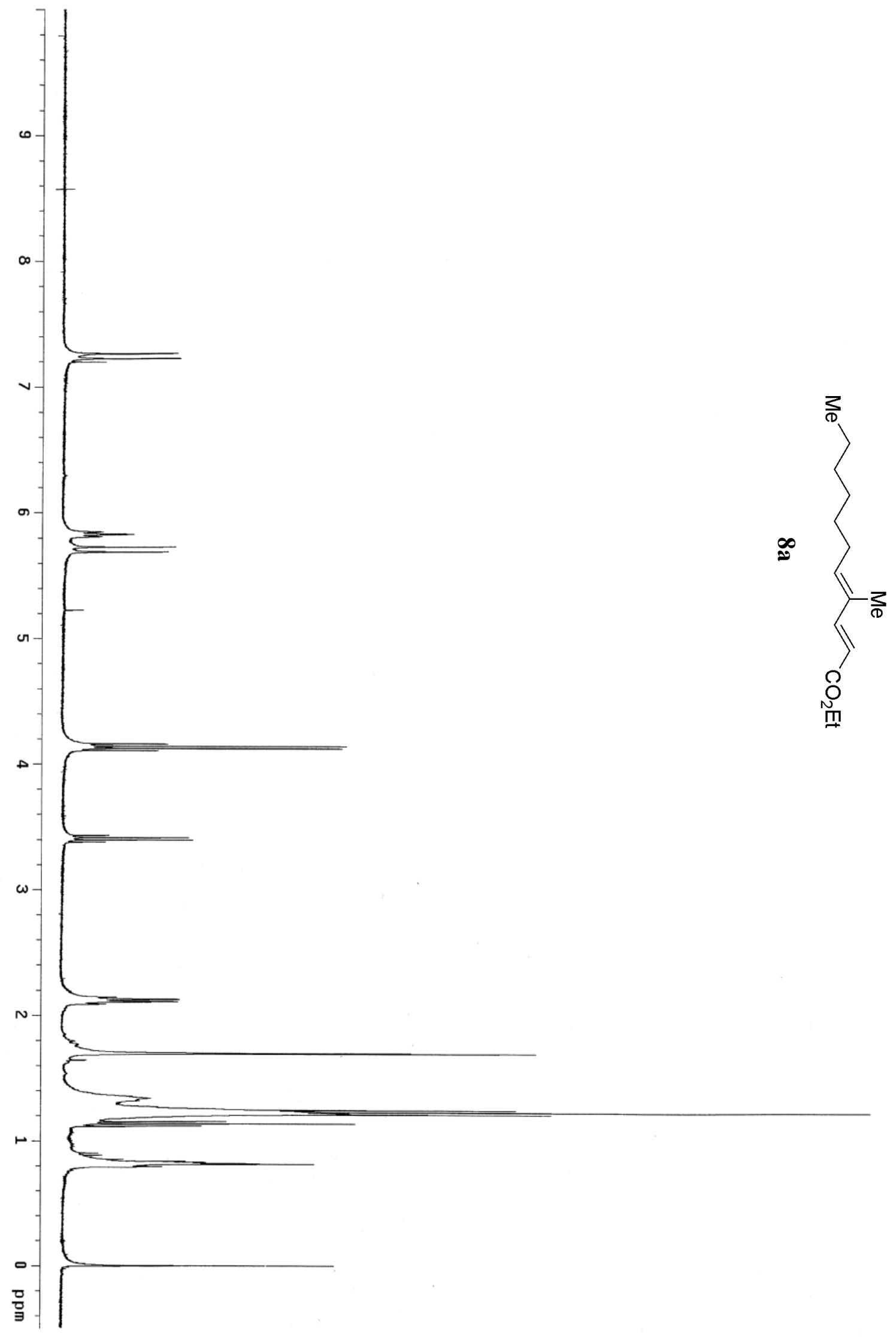




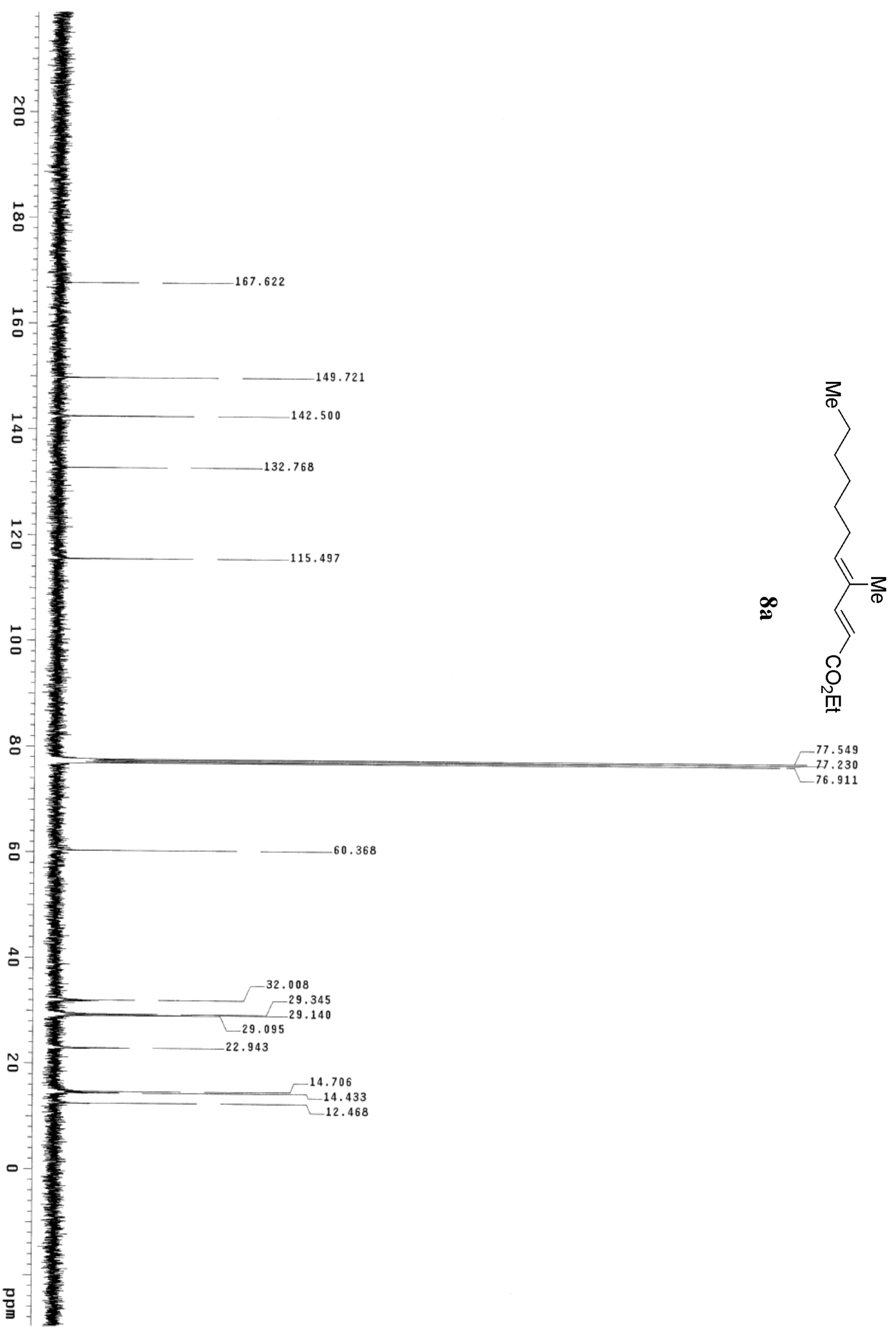




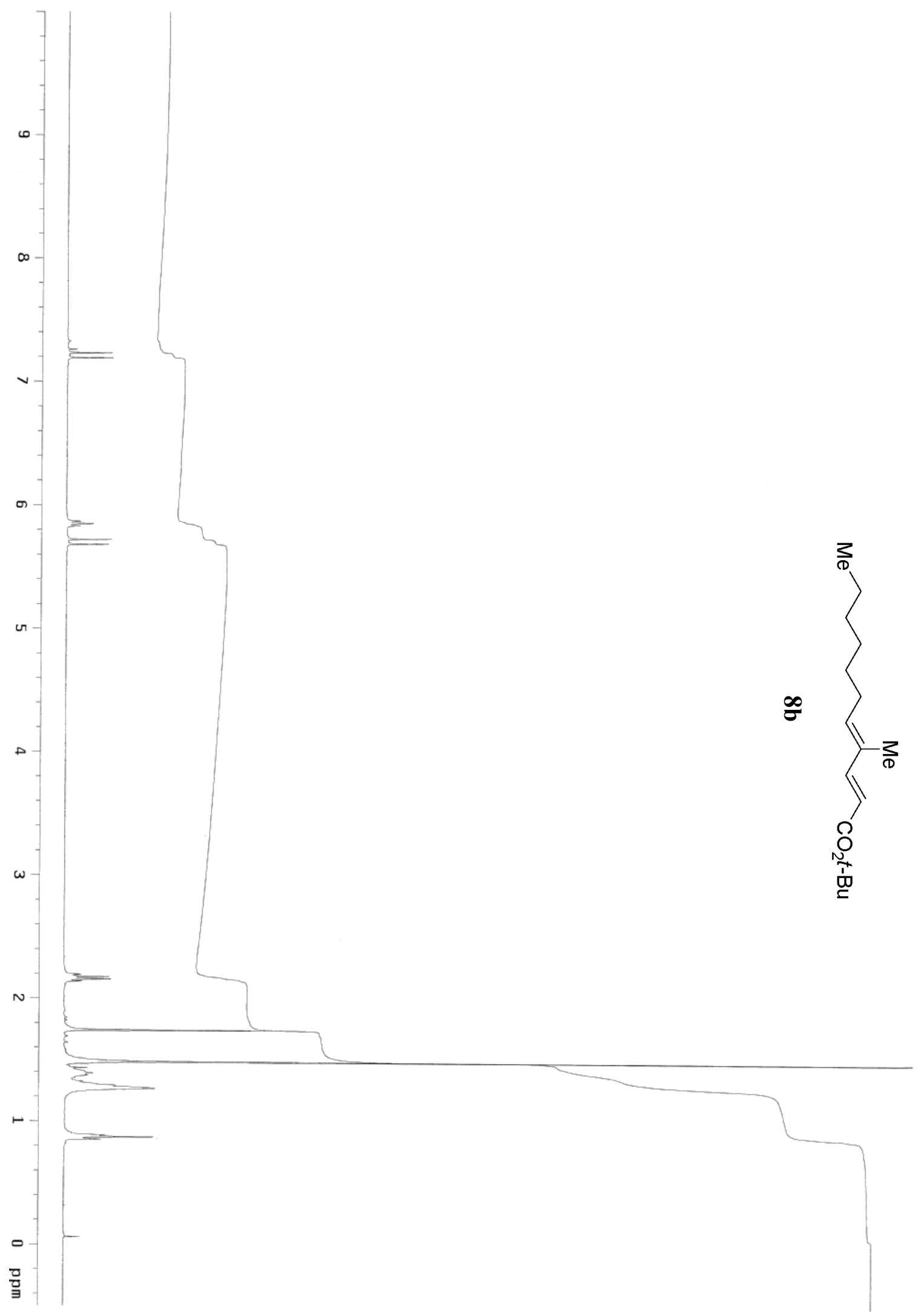




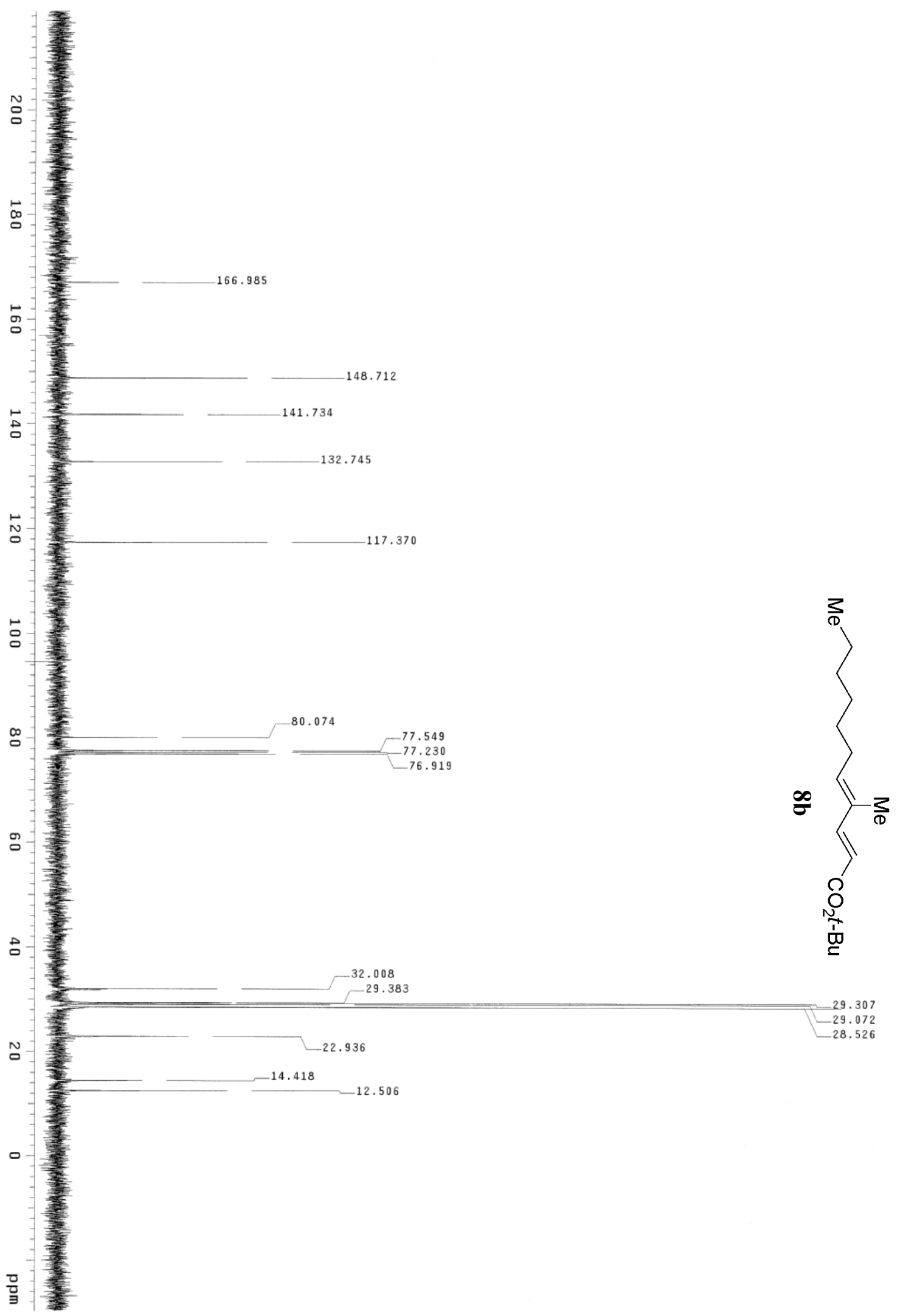




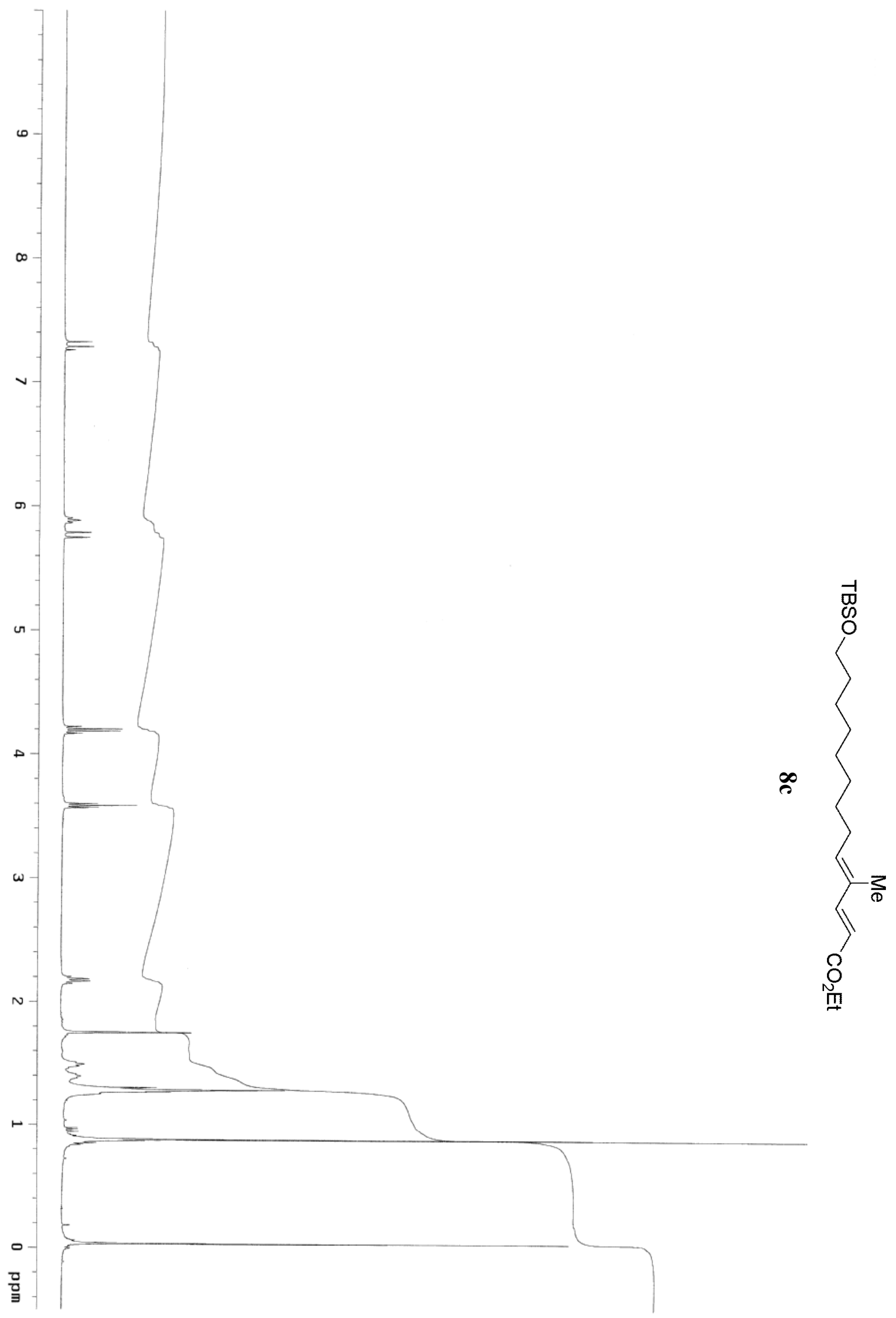

- S10 - 


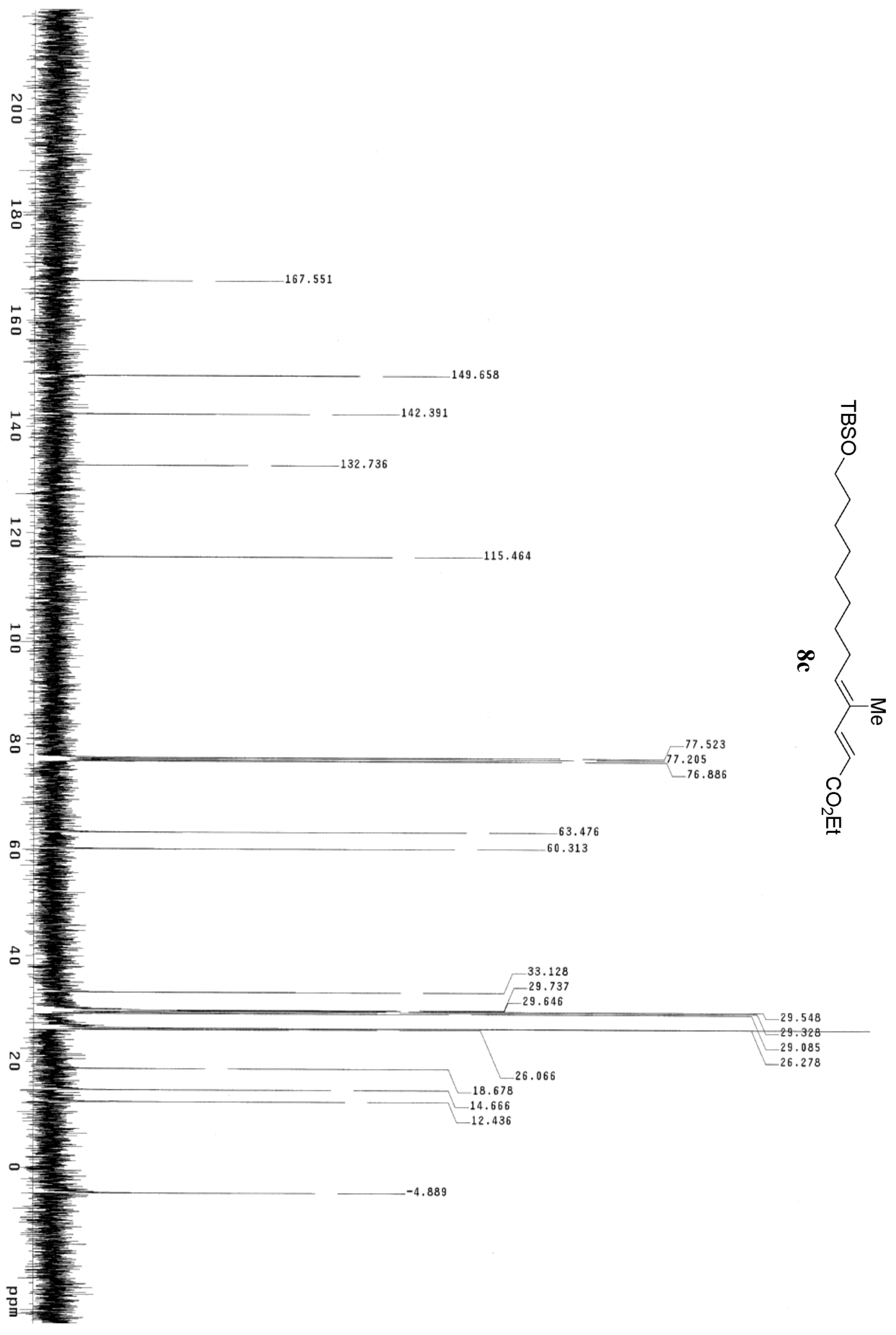




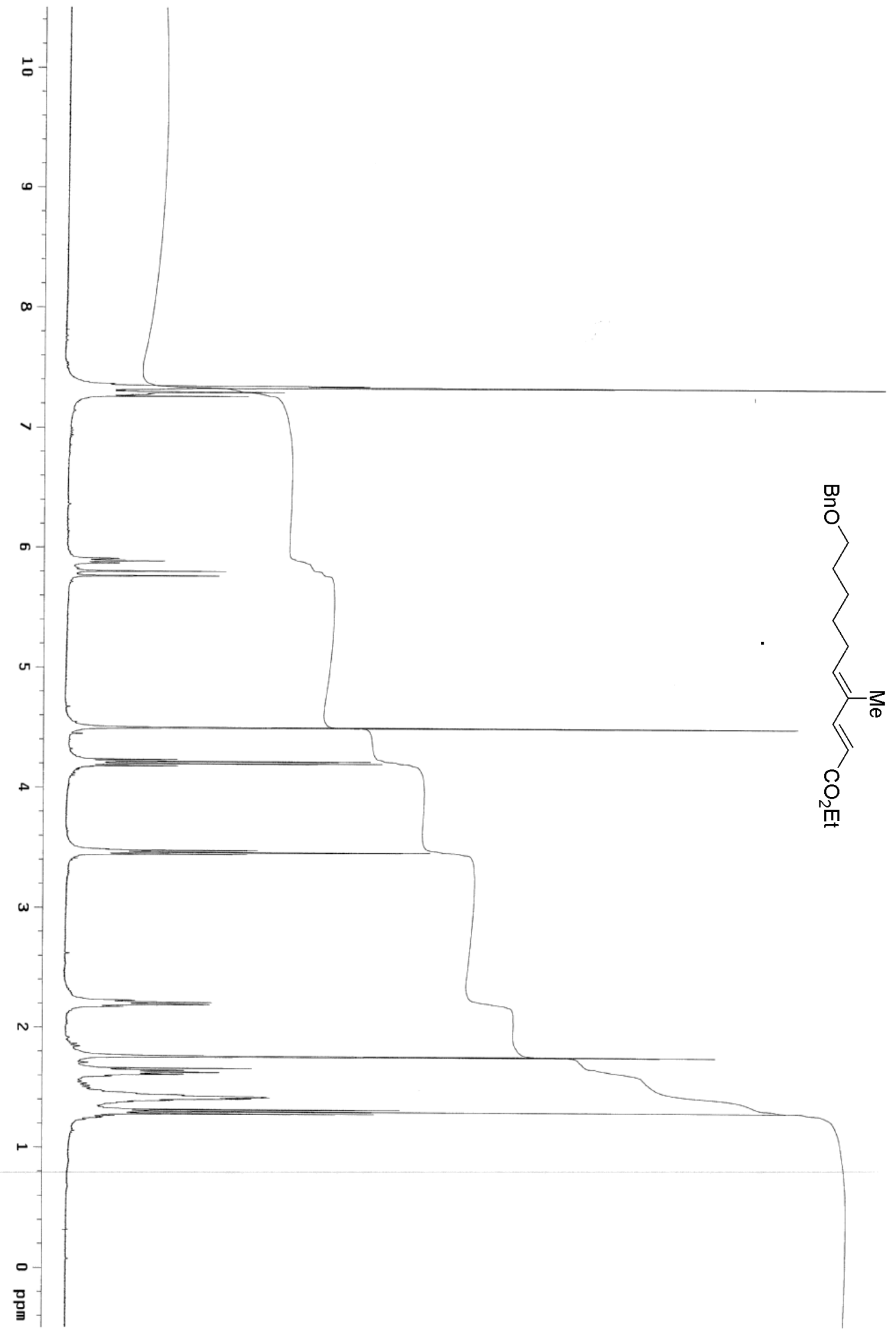

- S12 - 


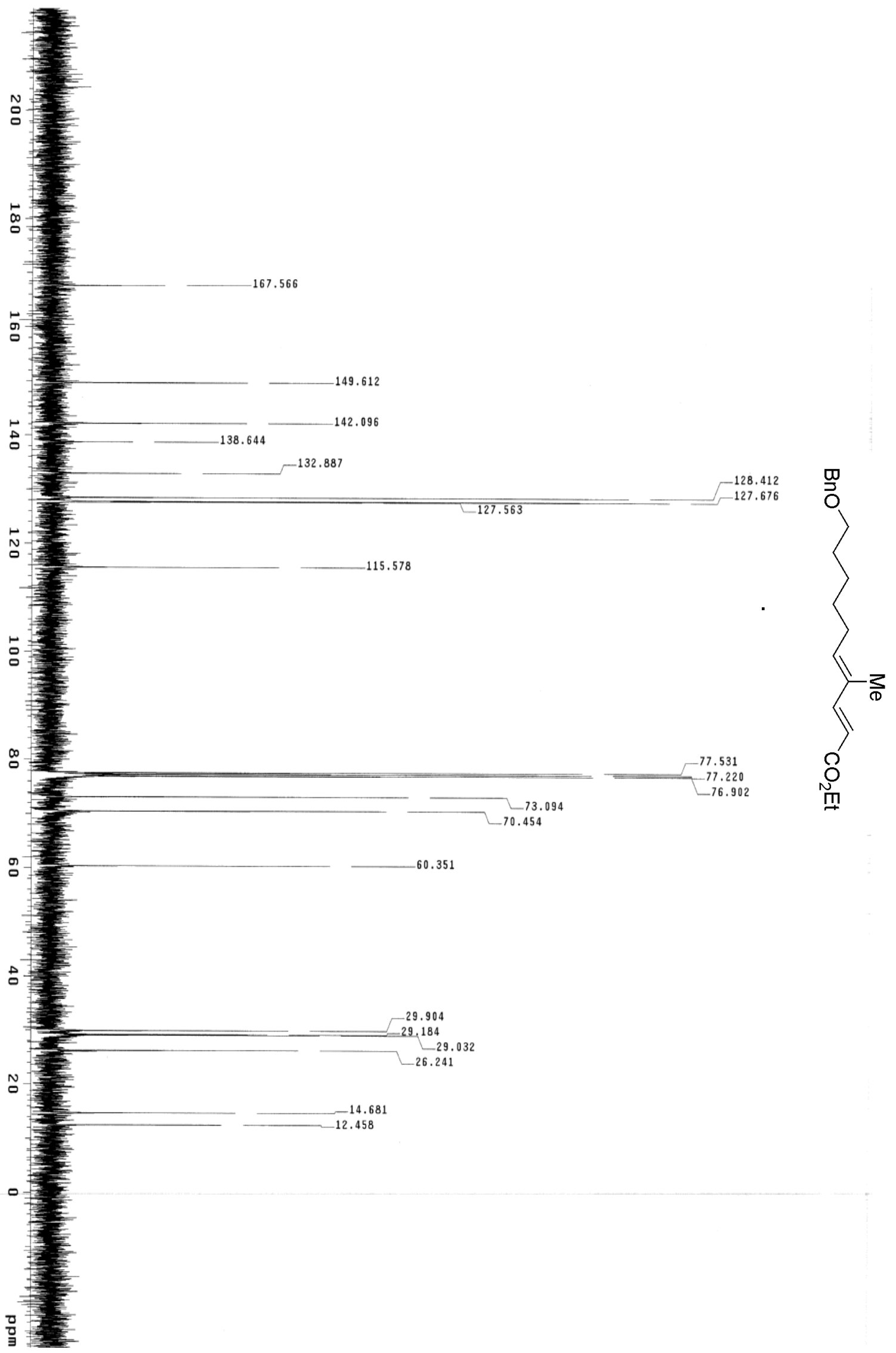




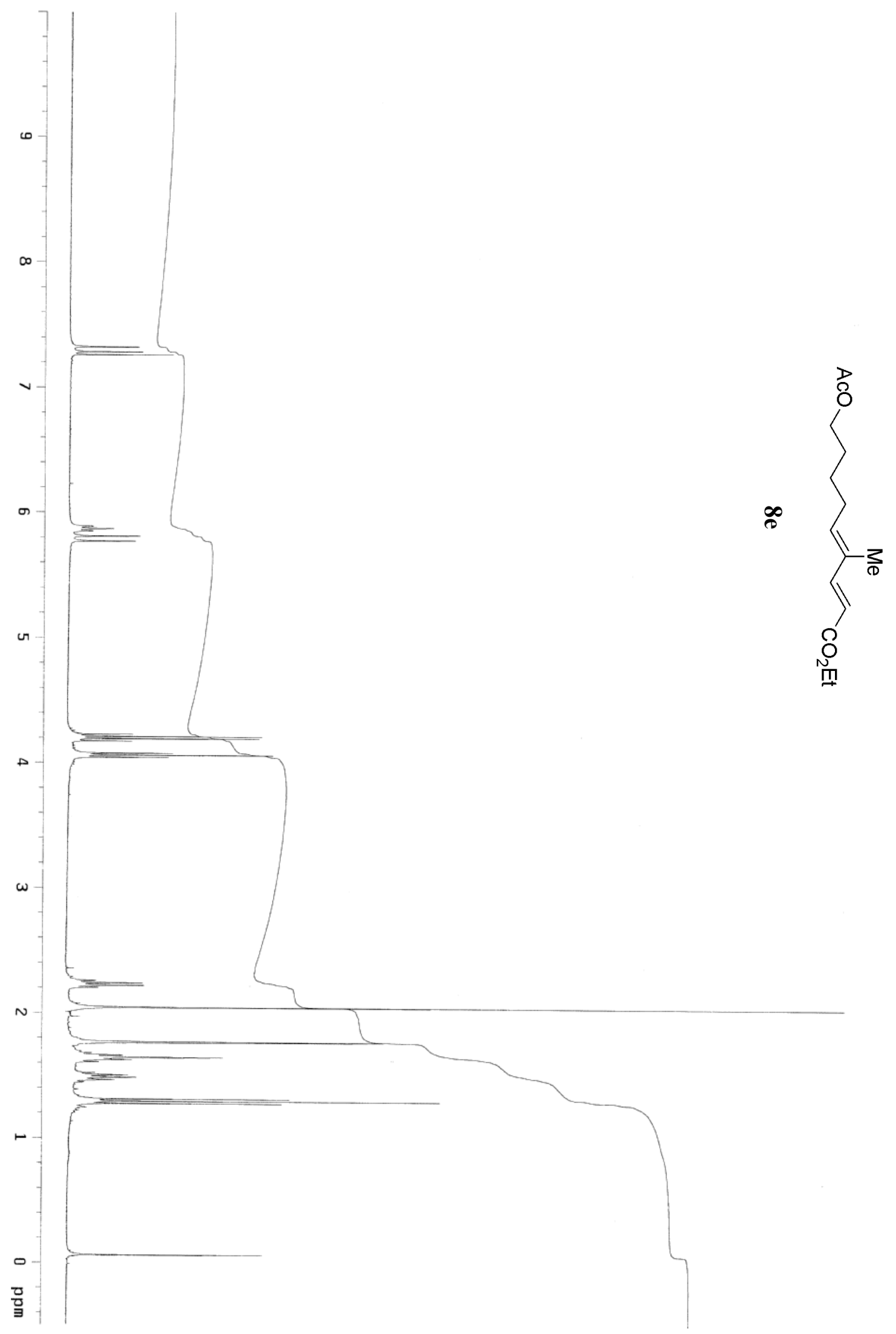

- S14 - 


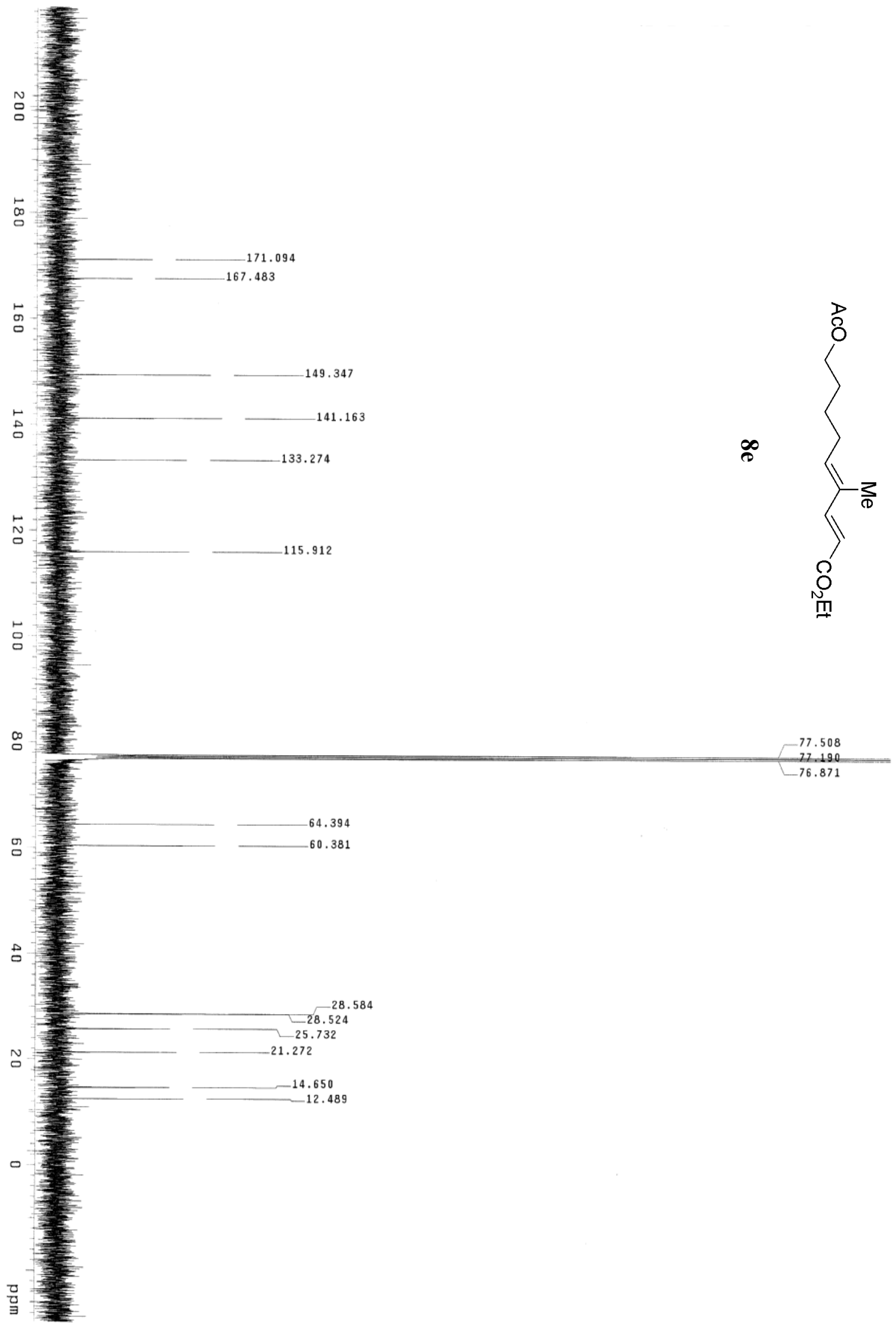

$-\mathrm{S} 15-$ 


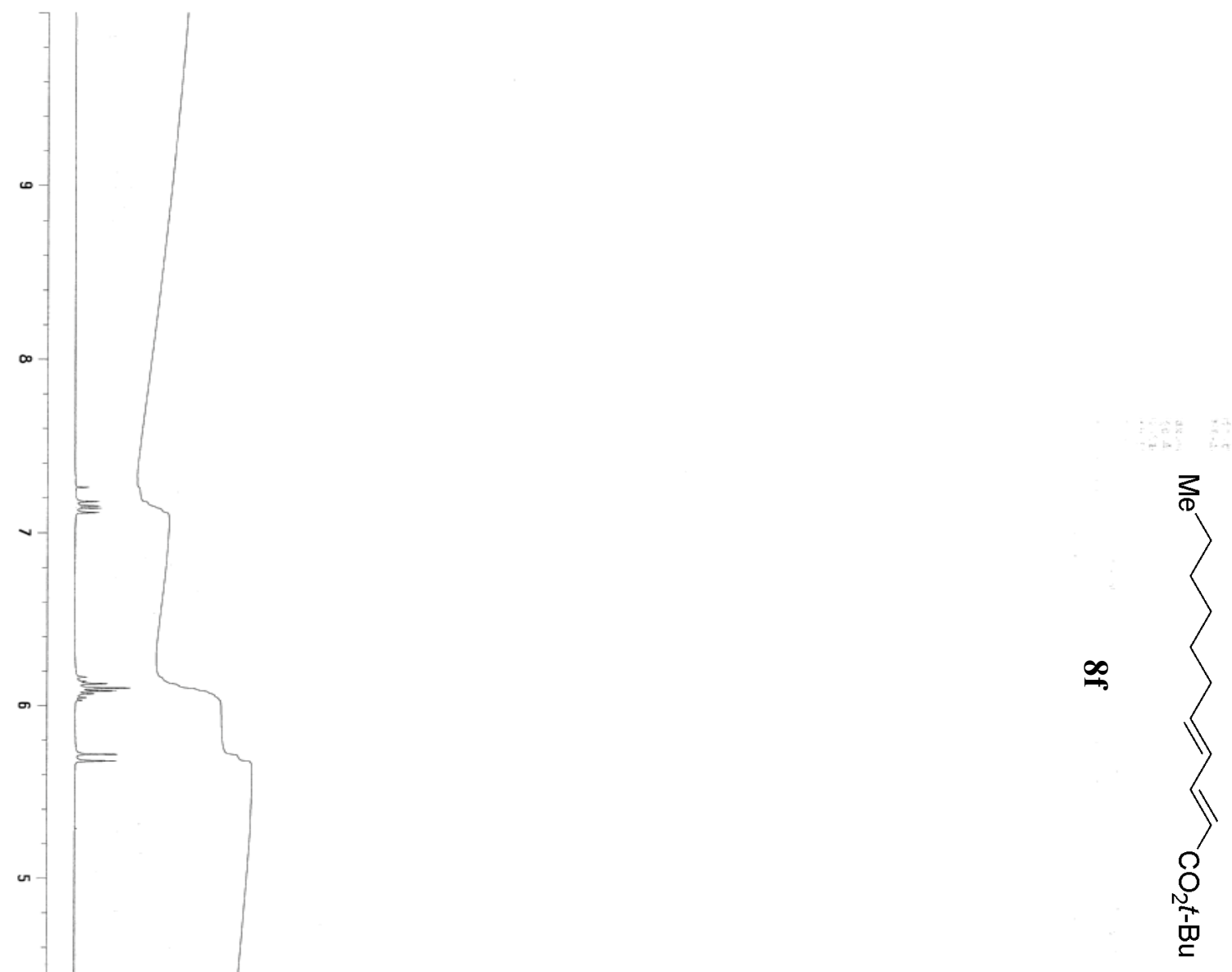

뭅 


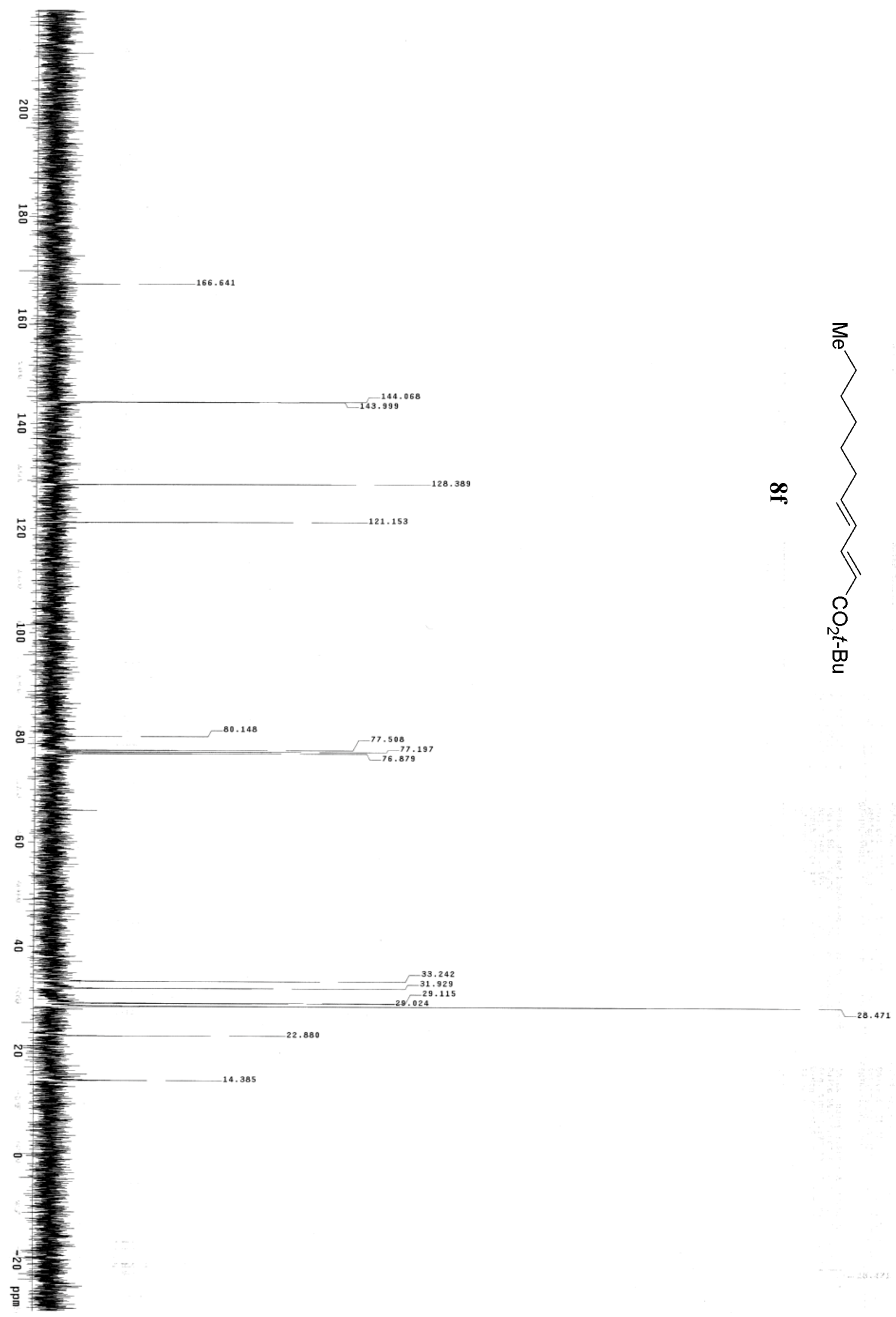




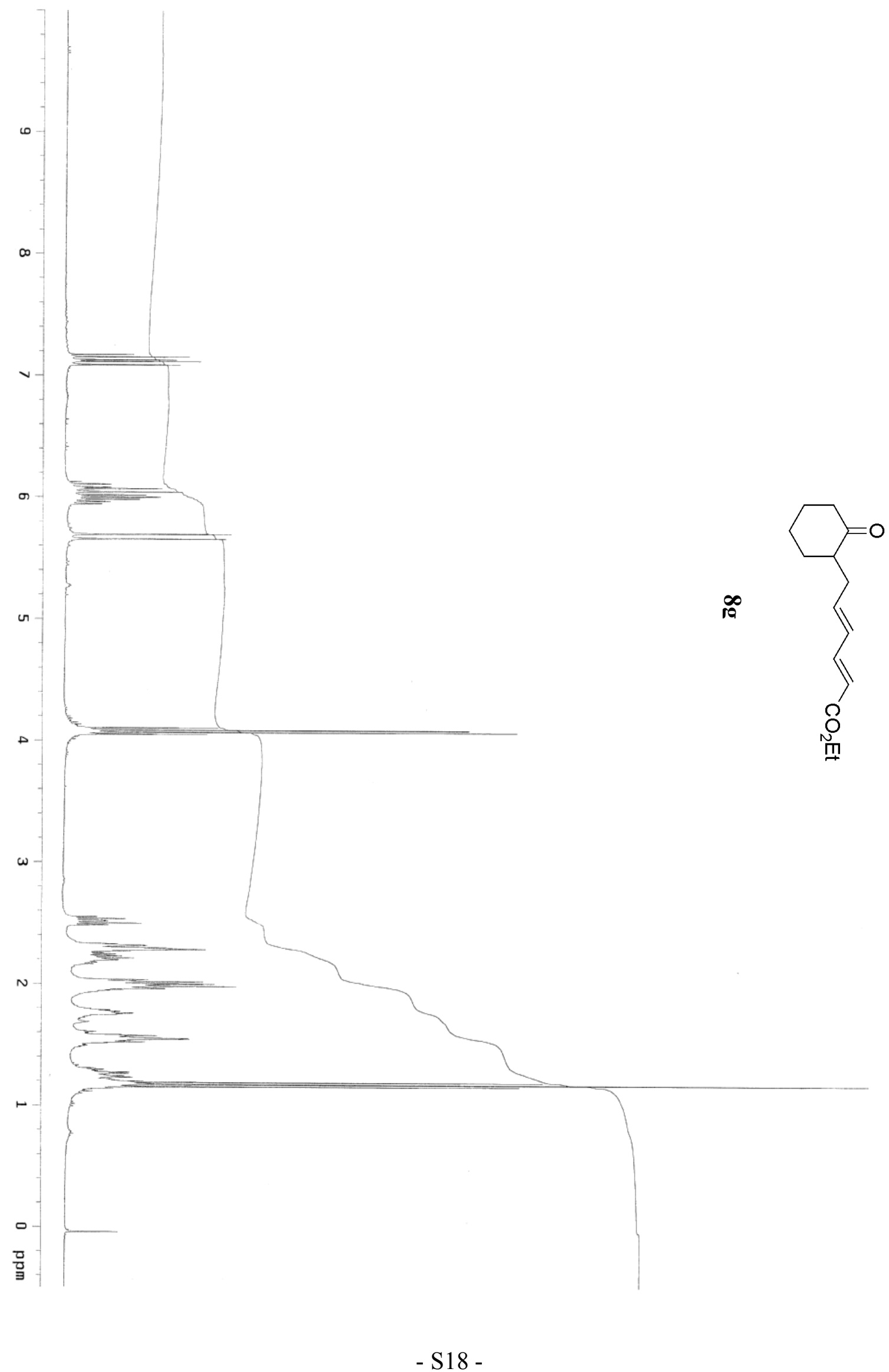




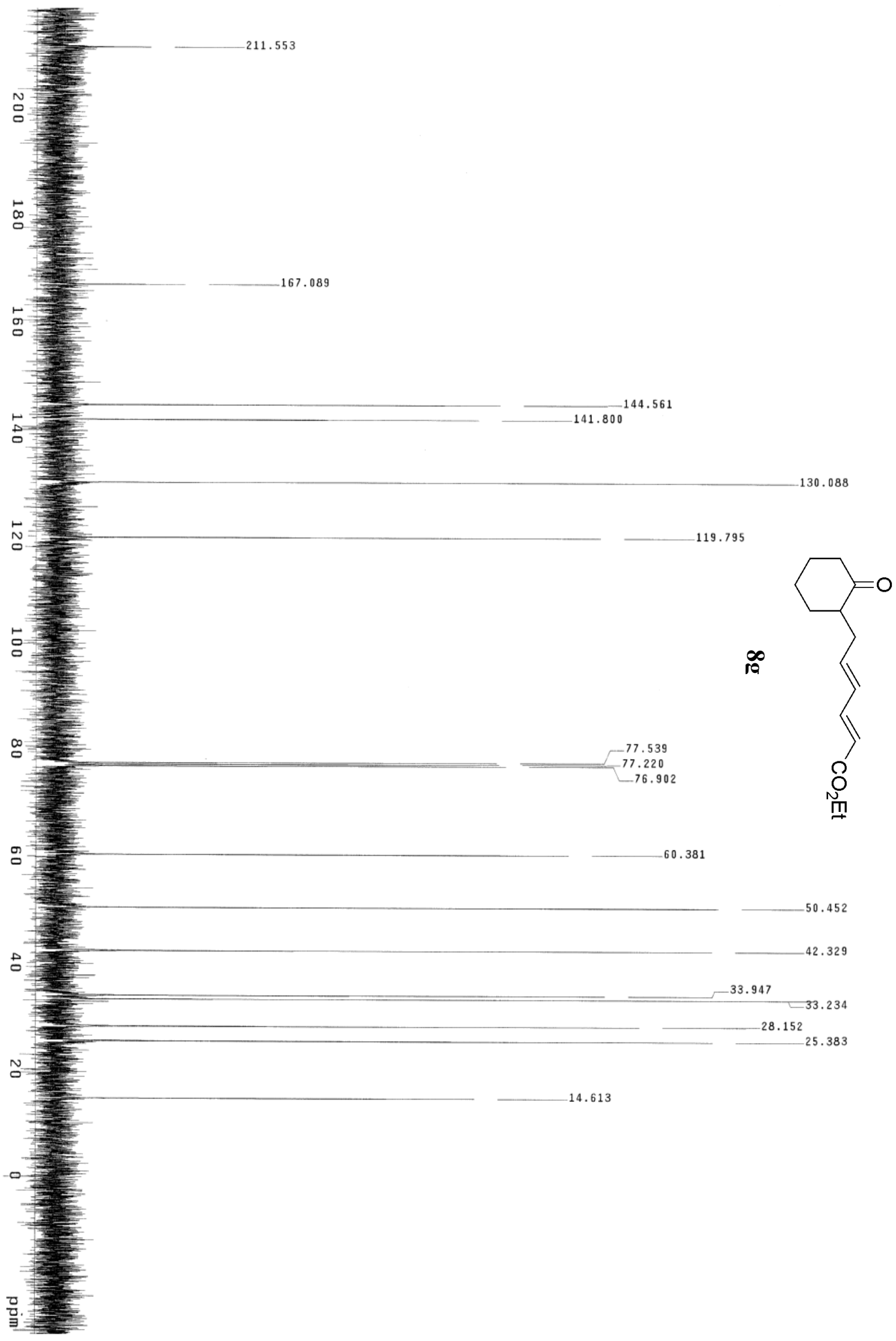




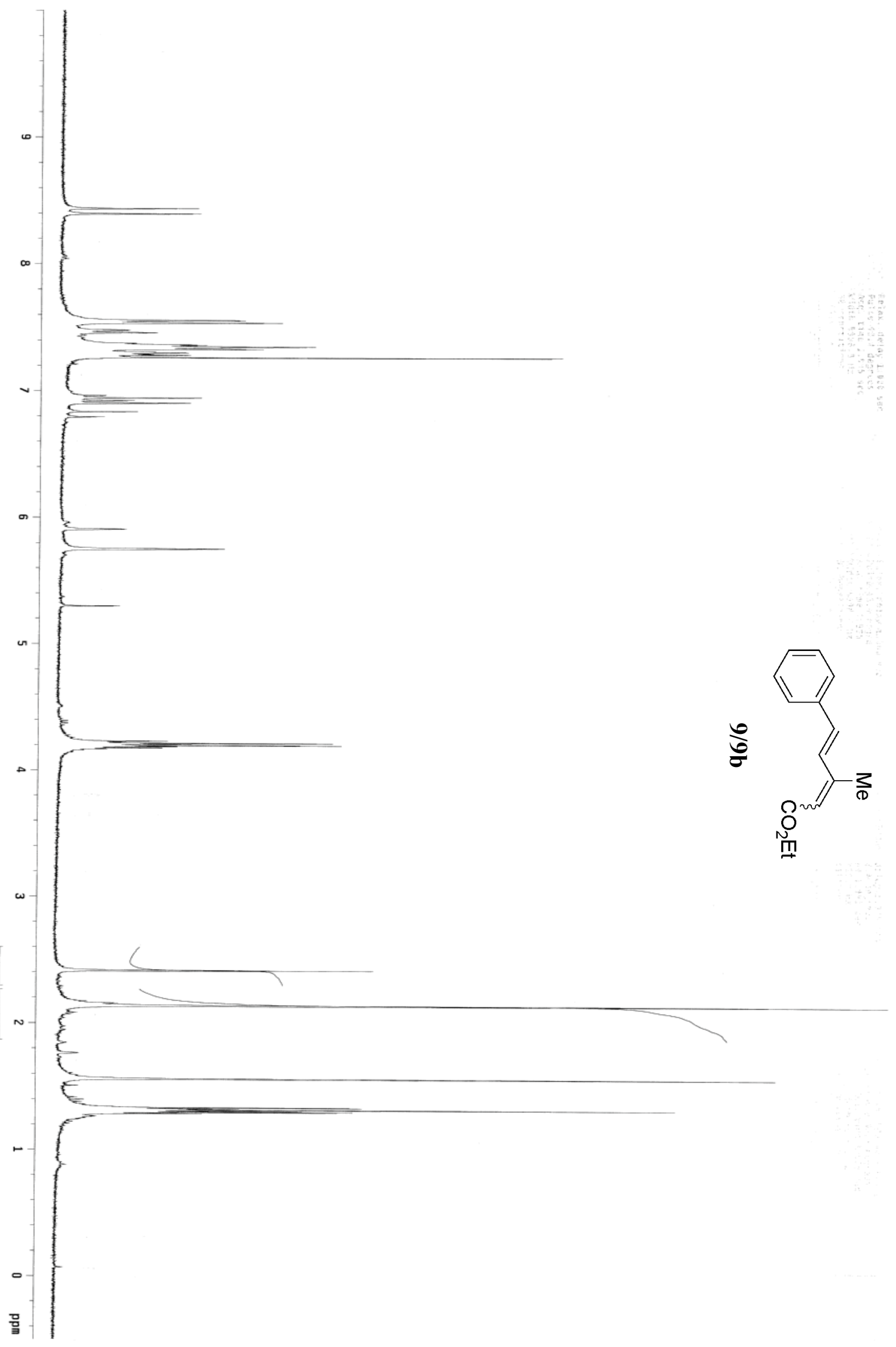

